\title{
Uso empírico de plantas medicinais para tratamento de diabetes
}

\author{
SANTOS, M.M. ${ }^{1}$; NUNES, M.G.S. ${ }^{2}$; MARTINS, R.D. ${ }^{3 *}$ \\ ${ }^{1}$ Universidade Federal de Pernambuco, UFPE, Rua Professor Nelson Chaves, s/n, Cidade Universitária, CEP: \\ 50670-420, Recife-Brasil mikaellacoby@hotmail.com ${ }^{2}$ Escola Santa Mônica, Avenida Severino Pinheiro, 12, \\ CEP: 55700-000, Limoeiro-Brasil marilia gabrielle170@hotmail.com ${ }^{3}$ Núcleo de Nutrição, UFPE/CAV, Universidade \\ Federal de Pernambuco, Rua Alto do Reservatório, s/n, CEP: 55.608-680, Vitória de Santo Antão-Brasil \\ *reneduarte@ig.com.br
}

\begin{abstract}
RESUMO: Diabetes é alvo interessante para a busca de novos métodos de tratamento com a possibilidade de uso de várias espécies de plantas medicinais. Este trabalho objetivou descrever a prevalência do uso de plantas medicinais consideradas hipoglicemiantes por pacientes diabéticos em Vitória de Santo Antão. O estudo foi do tipo descritivo transversal realizado com 158 pacientes diabéticos atendidos pelo programa HIPERDIA nos PSF's da cidade entre julho de 2009 a maio de 2010 , com a coleta de dados realizada por meio de formulário estruturado. Entre os entrevistados, $36 \%$ relatavam uso de plantas medicinais consideradas hipoglicemiantes. Foram citadas 35 plantas diferentes pertencentes à 24 famílias, sendo as mais freqüentes: Asteraceae $(12,5 \%)$ e Myrtaceae $(9,37 \%)$. A planta medicinal mais prevalente foi a pata de vaca (Bauhinia sp), com 16,8\%, seguida por azeitona roxa (Syzygium jambolanum DC.) e insulina (Cissus sicyoides L.). A maioria dos indivíduos (58\%) cultivava a planta medicinal que usavam e, entre aqueles que adquiriam, a principal fonte foi a de raizeiros $(28,16 \%)$.
\end{abstract}

Palavras-chave: Diabetes mellitus, plantas medicinais, fitoterapia, PSF - Hiperdia

ABSTRACT: Empirical use of medicinal plants for diabetes treatment. Diabetes is an attractive target to search for new methods of treatment, with the possibility of using several medicinal plant species. This study aimed to describe the prevalence of the use of medicinal plants considered hypoglycemic for diabetic patients from Vitoria de Santo Antão-Pernambuco State, Brazil. This was a transversal descriptive study conducted with 158 diabetic patients enrolled in the program HIPERDIA at the PSF's of this city, between July 2009 and May 2010 with data collected by means of structured form. Among interviewees, $36 \%$ reported the use of medicinal plants considered hypoglycemic. A total of 35 different plants belonging to 24 families were cited and the most frequent species were: Asteraceae (12.5\%) and Myrtaceae (9.37\%). The most prevalent medicinal plant was "pata-de-vaca" (Bahuinia sp.), with 16.8\%, followed by "azeitona roxa" (Syzygium jambolanum DC.) and "insulina" (Cissus sicyoides L.). Most individuals (58\%) cultivated the medicinal plant they used, and for those who acquired them, the main source was "raizeiros" [people similar to healers but who only sell medicinal plants] (28.16\%).

Key words: Diabetes mellitus, medicinal plants, phytotherapy, PSF - Hiperdia

\section{INTRODUÇÃO}

Um estudo multicêntrico sobre prevalência de Diabetes mellitus no Brasil apontou índice de 7,6\% na população brasileira entre 30-69 anos, atingindo cifras próximas a $20 \%$ na população acima dos 70 anos. Cerca de $50 \%$ dessas pessoas desconhecem o diagnóstico e $25 \%$ da população diabética não fazem nenhum tratamento (Paiva et al., 2006). Projeções da OMS mostraram que há em média 220 milhões de pessoas no mundo com diabetes, sendo que este número provavelmente dobrará entre 2005 e 2030 (Cecílio et al., 2008; WHO, 2009).

No tratamento do diabetes, os recursos medicamentosos são empregados, geralmente, em um segundo momento da terapêutica, quando da incapacidade de se controlar os níveis glicêmicos preferencialmente através da prática da dieta e de exercícios físicos. Entre os agentes medicamentosos disponíveis para a terapia do diabetes estão incluídos

Recebido para publicação em 27/12/2010

Aceito para publicação em 31/07/2011

Rev. Bras. PI. Med., Botucatu, v.14, n.2, p.327-334, 2012. 
a insulina e os hipoglicemiantes orais (principalmente, biguanidas e sulfoniluréias) (Assunção et al., 2002).

Os custos diretos para o atendimento ao diabetes variam de 2,5 a $15 \%$ dos gastos nacionais em saúde, dependendo da prevalência local de diabetes e da complexidade do tratamento disponível; portanto, a prevenção do diabetes e das complicações é a prioridade de saúde pública devido à elevada morbimortalidade associada (Ministério da Saúde, 2006).

Diversas espécies vegetais vêm sendo citadas na literatura como adjuvantes no tratamento da Diabetes mellitus atuando, tanto no tratamento da doença em si como atenuando seus sintomas e possíveis consequências e, desta forma, inúmeros estudos vêm sendo desenvolvidos com o objetivo de comprovar o efeito de espécies vegetais muitas vezes utilizadas apenas com base em dados empíricos. $\mathrm{O}$ Diabetes mellitus, por ser doença crônica, de tratamento contínuo, é alvo interessante para a busca de novos métodos de tratamento com a possibilidade de uso de várias espécies de plantas medicinais para o tratamento, contribuindo para triagens etnofarmacológicas e direcionamento de pesquisas que relacionem o potencial de espécies brasileiras para o tratamento desta condição patológica (Cecílio et al., 2008).

Já em 1975 o Conselho Nacional de Saúde referia-se à importância da medicina popular: " $A$ denominada -medicina popular- é constituída por práticas paralelas à -medicina oficial dominante-. Quem faz esta distinção é o sistema oficial de saúde, constatando sua existência e crescimento, a despeito de todo o avanço científico e tecnológico atual que tem o respaldo do saber científico e do sistema de produção (CNS, 1975)". Desta forma, a Organização Mundial da Saúde (OMS) reconhece a importância da fitoterapia sugerindo ser uma alternativa viável e importante também às populações dos países em desenvolvimento devido ao seu custo diminuído (Rezende \& Cocco, 2002).

No Brasil, o uso de terapias menos onerosas para o tratamento de enfermidades crônicodegenerativas representa ganho importante nos investimentos humanos e financeiros empregados na área de saúde. Assim, os fitoterápicos podem atuar como forma opcional de terapêutica levando em consideração o menor custo, e cujos benefícios adicionam-se aos da terapia convencional. Para tanto, são necessários maiores investimentos para estudos científicos nessa área de maneira a levar à comprovação da eficácia dessas espécies como hipoglicemiantes garantindo a segurança e eficácia na aplicação por parte dos profissionais de saúde (Borges et al., 2008).

Frente à crises vividas no setor saúde, o Ministério da Saúde, em 1994, implantou o Programa Saúde da Família (PSF), com o objetivo de proceder à reorganização da prática assistencial a partir da atenção básica, em substituição ao modelo tradicional de assistência, orientado para a cura de doenças. Assim sendo, o PSF pretende promover a saúde através de ações básicas que possibilitam a incorporação de ações programáticas de forma mais abrangente (DAB, 2000; MS, 2000). A dinâmica proposta pelo PSF, centrada na promoção da qualidade de vida e intervenção nos fatores que a colocam em risco, permite a identificação mais acurada e melhor acompanhamento dos indivíduos diabéticos e hipertensos (Paiva, 2006).

Visando o acompanhamento constante e devido ao aumento dos agravos em pacientes portadores de doenças cardiovasculares foi criado em 2002 um Plano de Reorganização da Atenção à Hipertensão Arterial e Diabetes mellitus, sendo este, denominado Hiperdia. Este programa objetiva atacar a fundo estes agravos estabelecendo metas e diretrizes para ampliar ações de prevenção, diagnóstico, tratamento e controle dessas patologias, através da reorganização do trabalho de atenção à saúde das unidades da rede básica dos Serviços de Saúde/Sistema Único de Saúde (SUS) (Brasil, 2003).

Neste estudo, objetivou-se descrever a prevalência do uso de plantas medicinais consideradas hipoglicemiantes por pacientes diabéticos no município de Vitória de Santo AntãoPE e comparar este conhecimento etnofarmacológico com estudos descritos na literatura científica.

\section{MATERIAL E MÉTODO}

\section{Caracterização do local estudado}

O presente trabalho foi realizado no município de Vitória de Santo Antão - PE, zona da mata sul do estado de Pernambuco, cuja população é de aproximadamente 126.399 mil habitantes, distribuídos numa área de aproximadamente $371,8 \mathrm{~km}^{2}$. Apresenta cobertura de Programa de Saúde da Família (PSF) de $91,9 \%$, em ampliação, contando atualmente com 28 unidades de Saúde da Família (24 PSF's e 4 PACs), cuja implementação do programa Hiperdia encontra-se em fase de conclusão.

\section{Período e estruturação do estudo}

O estudo foi do tipo descritivo transversal e ocorreu entre os meses de julho de 2009 a maio de 2010. O projeto foi aprovado pelo comitê de ética de pesquisa em seres humanos da Universidade Federal de Pernambuco (registro no 326/09), sendo enviada cópia e parecer compatível à secretaria de saúde do município de Vitória de Santo Antão - PE. Os pacientes assinaram voluntariamente um termo de consentimento livre e esclarecido, declarando conhecimento sobre a utilização dos dados obtidos 
e cientes da garantia de omissão de dados de identificação.

Foi realizado levantamento de dados em todos os postos de saúde da cidade a partir de dados do programa Hiperdia fornecidos pela Secretária Municipal de Saúde (SMS) do município, obtido o número de pacientes exclusivamente diabéticos atendidos pelo programa no município, e realizado as identificações e PSF's de origem. Em visita aos PSF's para localização dos pacientes procedeu-se a atualização dos dados fornecidos pela SMS. A coleta dos dados foi realizada mediante entrevista direta com os pacientes em visitas domiciliares e, para tanto, foi utilizado como instrumento, a entrevista semiestruturada (com dados sócio demográficos e referentes ao uso e aquisição de plantas medicinais e/ou fitoterápicos), complementada por diário de campo preenchido após cada entrevista com o objetivo de facilitar a análise dos dados. Na ocasião, e sempre que possível, procedeu-se à coleta de amostras das plantas medicinais e/ou fitoterápicos e/ou imagens (através de câmera fotográfica).

O estudo foi desenvolvido com todos os diabéticos que atenderam aos seguintes critérios de inclusão: 1) Idade superior a 18 anos; 2) Cadastrados no programa Hiperdia; 3) Não possuírem hipertensão arterial sistêmica; 4) Informar se faz ou fez uso de chás, macerações ou quaisquer outros preparados de plantas para fins medicinais ou agentes fitoterápicos para tratamento da Diabetes mellitus.

As plantas medicinais relatadas foram associadas na pesquisa aos nomes científicos mais prevalentes relatados na literatura, considerando-se em tal classificação, a denominação popular associada ao uso como agente hipoglicemiante.

Os dados foram plotados e analisados no programa Epi.info 6.04, através da distribuição em médias e proporções e comparação entre médias através dos testes ANOVA e T de Student e comparação entre proporções realizada por teste do Qui-quadrado $\left(X^{2}\right)$.

\section{RESULTADO}

O universo da pesquisa constou de 158 pacientes exclusivamente diabéticos. A caracterização da população estudada revelou predomínio de pacientes do sexo feminino, com $63,5 \%$ $(p<0,05)$ e prevalência de idades entre 51 e 60 anos. Quanto ao perfil profissional, a maioria dos pacientes referia como ocupação "atividades do lar" (61,4\%), com nível de escolaridade prevalente o ensino fundamental incompleto $(56,1 \%)$. A presença de qualquer tipo de alergia era desconhecida por $93 \%$ da população estudada. Com relação à patologia, 0 tempo de diagnóstico do diabetes variou entre 1 a 5 anos na maioria dos pacientes $(49,1 \%)$. Dentre os entrevistados, $36,5 \%$ referiram ao uso de algum tipo de planta medicinal ou fitoterápico no tratamento do Diabetes mellitus. Foi observado que o PSF onde houve o maior número de entrevistas foi aquele do bairro Caiçara (13,29\%), o PSF de maior prevalência quanto ao uso de plantas medicinais foi o posto do bairro Jardim Ipiranga (83,3\%), seguido de Cajueiro $(66,6 \%)$ e Redenção (60\%). Nenhum paciente referia abandonar a farmacoterapêutica em detrimento do uso de plantas medicinais, sendo este tratamento complementar àquele.

Foram citadas 35 plantas diferentes com possível ação hipoglicemiante, pertencentes a 24 famílias, sendo as mais freqüentes: Asteraceae $(12,5 \%)$ e Myrtaceae (9,37\%). As plantas medicinais mais prevalentes foram pata-de-vaca (Bahuinia $\mathrm{sp}, 16,8 \%$ ), azeitona roxa (Syzygium jambolanum DC., $15,88 \%$ ) e insulina (Cissus sicyoides L., 14,01\%), conforme representado na Tabela 1.

A maioria dos indivíduos (58\%) cultivava a planta medicinal de uso, enquanto $25 \%$ adquiriam de terceiros, sendo os raizeiros a principal fonte $(28,16 \%)$. As partes mais utilizadas foram as folhas $(64 \%)$ e entrecascas (14\%), conforme representado na Figura 1. Quanto a forma de preparo, tivemos: decocção (69\%), infusão (12\%) e tintura (9\%), e quanto ao tempo de utilização do chá em até $24 \mathrm{~h}$ $(56 \%)$. Sobre à forma de armazenamento foi prevalente a refrigeração em geladeira doméstica (61\%). Sobre a posologia das preparações medicinais foi observado que a maior parte dos entrevistados fazia uso dos chás entre $1-4$ vezes ao dia $(65,51 \%)$ e $45 \%$ revelaram ter recebido indicação de uso da planta medicinal por amigos. Com relação ao reconhecimento das plantas medicinais/fitoterápicos, 51,6\% responderam que reconheciam a planta através de suas folhas, enquanto $15 \%$ não reconheciam a planta que utilizavam, pois adquirem de terceiros ou recebem por doação de algum conhecido.

A maioria dos entrevistados desconhecia qualquer efeito colateral ou contra indicação das plantas medicinais utilizadas (88\%), sendo as mais freqüentes citações descritas na Tabela 2, com poucos relatos de queixas relacionadas ao uso das preparações (9\%).

\section{DISCUSSÃO}

Não se deve subestimar o conhecimento empírico do homem primitivo. Desde o seu primórdio o homem explora a natureza, principalmente plantas e animais para se alimentar, medicar, construir abrigos e roupas. As plantas têm sido utilizadas pela humanidade como medicamentos desde os primórdios. Supõe-se que mais de $70 \%$ dos medicamentos derivados de plantas foram desenvolvidos com base no conhecimento folclórico.

Rev. Bras. PI. Med., Botucatu, v.14, n.2, p.327-334, 2012. 
TABELA 1. Prevalência de plantas medicinais com relato de atividade hipoglicemiante utilizadas por pacientes diabéticos no município de Vitória de Santo Antão-PE.

\begin{tabular}{|c|c|c|c|c|}
\hline Família & Nome científico & Nome popular & (\%) & Referências \\
\hline Fabaceae & Bahuinia sp & Pata de vaca & 16,82 & $\begin{array}{l}\text { Pepato et al., 2002; Lino et al., } \\
\text { 2004; Fuentes et al., 2004; } \\
\text { Cecílio et al.,2008. }\end{array}$ \\
\hline Myrtaceae & Syzygium jambolanum DC. & Azeitona roxa & 15,88 & $\begin{array}{l}\text { Grover et al., 2000; Soares et al., } \\
2000 ; \text { Zanoello et al., 2001; } \\
\text { Rao et al., 2001; Rathi et al., 2002; } \\
\text { Prince et al., 2003. }\end{array}$ \\
\hline Vitaceae & Cissus sicyoides L. & Insulina & 14,01 & $\begin{array}{l}\text { Pepato et al., 1998; } \\
\text { Carvalho et al., } 2005 .\end{array}$ \\
\hline Asteraceae & Cynara scolymus L. & Alcachofra & 5,6 & - \\
\hline Annonaceae & Annona Squamosa L. & Condessa & 4,67 & $\begin{array}{l}\text { Bragança, 1996; } \\
\text { Shirwaikar et al., } 2004 .\end{array}$ \\
\hline Anacardiaceae & Anacardium occidentale L. & Caju roxo & 3,73 & Arduíno \& Soares (1951). \\
\hline Caesalpiniaceae & Tamarindus sp & Tamarindo & 3,73 & - \\
\hline Passifloraceae & Passiflora spp & Maracujá & 2,8 & $\begin{array}{l}\text { Córdova et al., } 2005 \text {; } \\
\text { Krahn et al., } 2008\end{array}$ \\
\hline Myrtaceae & Punica granatum L. & Romã & 2,8 & Jafri et al., 2000. \\
\hline Malvaceae & Malva spp & Malva branca & 1,86 & - \\
\hline \multirow[t]{2}{*}{$\begin{array}{l}\text { Chrysobalanaceae } \\
\text { Sapotaceae }\end{array}$} & $\begin{array}{l}\text { Licania rigida Benth. } \\
\text { Sideroxylon obtusifolium }\end{array}$ & Oiticica & 1,86 & - \\
\hline & (Roem. \& Schult.) T.D. Penn. & Quixaba & 1,86 & $\begin{array}{l}\text { Almeida et al, 1982; } \\
\text { Naik et al., 1991; }\end{array}$ \\
\hline \multirow[t]{2}{*}{ Labiatae } & Plectranthus barbatus Andr. & Sete dores & & \\
\hline & & Malva-santa & 1,86 & - \\
\hline Piperaceae & Peperomia pellucida (L.) Kunth & Língua de sapo & 1,86 & - \\
\hline Asteraceae & Bidens pilosa L. & Picão & 1,86 & - \\
\hline Bignoniaceae & Crescentia cujete L. & Coité & 0,93 & - \\
\hline \multirow[t]{2}{*}{ Moraceae } & Artacarpus altilis (Park.) & & & \\
\hline & Forberg (A. communis) & Fruta pão & 0,93 & - \\
\hline Lamiaceae & Salvia aff. officinalis L. & Sálvia & 0,93 & - \\
\hline Solanaceae & Solanum gilo Raddi & Jiló & 0,93 & Silva et al., 1998. \\
\hline Apiaceae & Coriandrum sativum L. & Coentro & 0,93 & - \\
\hline Musaceae & Musa x paradisiaca L. & Banana prata & 0,93 & - \\
\hline Equisetaceae & Equisetum sp & Cavalinha & 0,93 & - \\
\hline Myrtaceae & Eugenia uniflora L. & Pitanga & 0,93 & - \\
\hline- & - & Pau de sapo & 0,93 & - \\
\hline \multirow[t]{2}{*}{ Cucurbitaceae } & Mormodica charantia L. & Melão de & & \\
\hline & & São Caetano & 0,93 & $\begin{array}{l}\text { Correa, 1984; } \\
\text { Marles \& Farnsworth, 1995; } \\
\text { Bragança, } 1996 .\end{array}$ \\
\hline Euphorbiaceae & Manihot esculenta Crantz. & Mandioca & 0,93 & - \\
\hline Lamiaceae & Ocimum urticifolium Roth. & Alfavaca & 0,93 & - \\
\hline Liliaceae & Allium sativum $\mathrm{L}$. & Alho & 0,93 & Baluchnejadmojarad et al., 2003; \\
\hline Costaceae & Costus sp & Cana de macaco & 0,93 & - \\
\hline Asteraceae & Artemisia absinthium L. & Losna & 0,93 & - \\
\hline Asteraceae & Egletes viscosa (L). Less & Marcela & 0,93 & - \\
\hline Solanaceae & Solanum melongena $\mathrm{L}$. & Berinjela & 0,93 & Derivi et al., 2002. \\
\hline Anacardiaceae & Mangifera indica L. & Manga espada & 0,93 & - \\
\hline \multirow[t]{2}{*}{ Misturas de ervas } & - & Garrafada* & 0,93 & - \\
\hline & - & Chá da vida** & 0,93 & - \\
\hline
\end{tabular}

*Garrafada constituída por misturas de ervas cujas espécies não eram conhecidas pelos usuários; **Mistura a base de insulina, pata de vaca, pau-ferro, pau-tenente, graviola, cana do brejo e berinjela. 


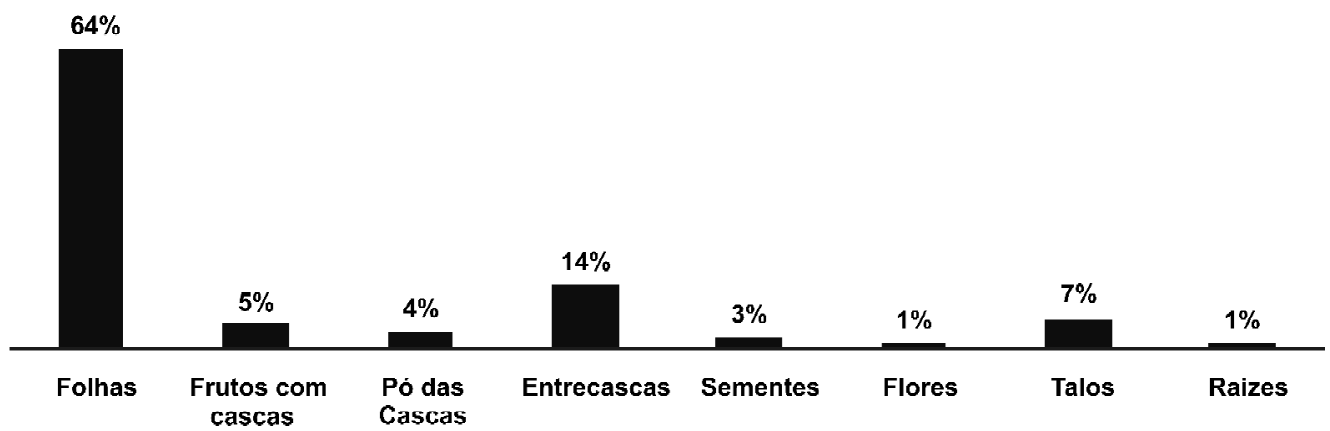

FIGURA 1. Prevalência de partes das plantas medicinais utilizadas por pacientes diabéticos.

TABELA2. Conhecimento dos pacientes diabéticos que utilizam plantas medicinais para tratamento desta patologia sobre efeitos colaterais das ervas

\begin{tabular}{|c|c|}
\hline Planta Medicinal & Efeito Colateral* \\
\hline $\begin{array}{l}\text { Pata de vaca (Bahuinia sp) } \\
\text { de vaca cega/deixa a vista embaçada" }\end{array}$ & "Só pode tomar quem é diabético, quem não é não pode tomar"'Pata \\
\hline $\begin{array}{l}\text { Condessa (Annona Squamosa L.) } \\
\text { mal" }\end{array}$ & “... a condessa deu agonia” "Uma amiga tomou demais e passou \\
\hline Insulina (Cissus sicyoides L.) & "...deixou a vista embaçada" "Não é pra tomar direto, nem muito forte" \\
\hline Azeitona roxa (Syzygium jambolanum DC.) & "Uma amiga tomou muito forte e teve alergia" \\
\hline
\end{tabular}

Na realidade, desde quando o homem apareceu neste planeta as primeiras explorações tecnológicas começaram, ocasionando o início do desenvolvimento econômico e do progresso social (Garcia, 1995).

Diegues (1996) afirma que o uso de plantas medicinais está relacionado à cultura popular que é transmitida de geração para geração nas comunidades tradicionais (ribeirinhas, indígenas, quilombolas, entre outros, e também nas populações contemporâneas). Enquanto Araújo (1977) informa que esta cultura é um dos agentes de solidariedade entre os membros da sociedade, dando até certo ponto uniformidade nos padrões de comportamento do grupo, orientando a conduta dos indivíduos (Macedo \& Ferreira, 2004), o que justifica o uso das plantas medicinais por indicação de amigos, entre os entrevistados. De fato, este é um hábito muito freqüente entre os usuários desta prática, visto que poucos médicos prescrevem plantas medicinais e fitoterápicos, ficando a atividade, geralmente, a cargo de curandeiros e raizeiros.

Em publicação do Museu Goeldi (Belém) sobre dados etnobotânicos de plantas medicinais da Amazônia, mais de 300 espécies de fitoterápicos foram relacionadas. Estas plantas são usadas popularmente contra dezenas de doenças infecciosas a parasitárias, vetores, problemas crônicodegenerativos, emagrecimento, regulação da menstruação, procedimento abortivo e até como antídoto ao veneno de cobra (Garcia, 1995).
No Brasil, o uso de terapias menos onerosas para o tratamento de enfermidades crônicodegenerativas representa importante ganho nos investimentos humanos e financeiros empregados na área de saúde. Assim, os fitoterápicos podem atuar como forma opcional de terapêutica levando em consideração seu menor custo, cujos benefícios adicionam-se aos da terapia convencional (Borges et al., 2008).

O Brasil possui a maior biodiversidade do planeta com cerca de $23 \%$ das espécies vegetais existentes em todo o mundo (Batalha et al.,2003) o que, associado à uma rica diversidade étnica e cultural, detém um valioso conhecimento tradicional associado ao uso de plantas medicinais e tem o potencial necessário para desenvolvimento de pesquisas que resultem em tecnologias e terapêuticas apropriadas (Ministério da Saúde, 2006). Esta biodiversidade do país contribuiu para que o uso das plantas medicinais fosse considerado um campo estratégico para o Brasil, cujo maior potencial econômico da biodiversidade está na descoberta de novas drogas derivadas diretamente ou sintetizadas a partir de recursos biológicos (Brito, 2010).

Resultados obtidos por Lino et al. (2004) apontam para a validade do uso clínico de Bauhinia forficata no tratamento do diabetes tipo 2. Segundo Cecílio et al. (2008), o decocto de $B$. forficata pode ser usado no tratamento do diabetes porque melhora a condição do diabetes sem causar toxicidade tecidual 
detectável. Pepato et al. (2002) demonstraram redução significativa da glicose na urina e no soro de ratos tratados com $B$. forficata. O extrato bruto de $B$. candicans apresentou atividade hipoglicêmica com redução da excreção urinária da glicose, sugerindo aumento do metabolismo da glicose. Pepato et al. (2004) ao administrarem doses de $200 \mathrm{mg} \mathrm{Kg}^{-1}$ e 400 $\mathrm{mg} \mathrm{Kg}^{-1}$ ao longo de sete dias de tratamento em ratos diabéticos e não diabéticos concluíram que a $B$. forficata pode reduzir glicose, triglicérides e colesterol total.

Os resultados encontrados por Soares et al. (2000) e Grover et al. (2000) que utilizaram respectivamente a folha e a semente da azeitona roxa demonstraram a ação hipoglicemiante da planta. Prince et al. (2003) demonstraram que a azeitona roxa (Syzygium jambolanum DC. ou Eugenia jambolana Lam.) possui propriedades antioxidantes, elevando a atividade das enzimas removedoras de radicais livres no fígado, rim e coração de ratos induzidos com aloxano e, desta forma, protege esses tecidos do estresse oxidativo gerado pela hiperglicemia diabética.

A Cissus sicyoides tem atividade farmacológica comprovada no tratamento de convulsão, doenças do coração (Moura, 1986; Elizabetsky, 1988; Costa, 1990) e controle de diabetes crônica (Pepato et al., 1998). A administração oral crônica do extrato hidroalcoólico de insulina (Cissus sicyoides L.) em ratos diabéticos, induzidos por estreptozotocina, promoveu uma melhora na glicemia refletida na redução da glicosúria. Em outro estudo, administrando-se o decocto das folhas da planta por períodos mais extensos em ratos normais e diabéticos observou-se redução significante no consumo de alimentos e fluidos e volume da urina, bem como também na glicemia, glicosúria e uréia, em comparação com animais controle.

Inúmeros outros estudos experimentais enfatizam a ação hipoglicemiante de plantas como a condessa - Annona squamosa L. (Bragança, 1996; Shirwaikar et al., 2004), caju roxo - Anacardium occidentale L. (Arduíno \& Soares, 1951), maracujá (Passiflora edulis Sims.) (Ramos, 2004; Córdova et al., 2005; Krahn et al., 2008), romã - Punica granatum L. (Nogueira \& Pereira, 1986; Jafri et al., 2000), quixaba Bumelia sartorum Mart. (Naik et al, 1991; Carvalho et al., 2005), jiló - Solanum gilo Raddi (Silva et al., 1998), melão de São Caetano - Momordica charantia L. (Marles \& Farnsworth, 1995), alho - Allium sativum L. e cebola - Allium cepa L. (Baluchnejadmojarad et al., 2003), berinjela - Solanum melongena (Derivi et al., 2002), dentre outras espécies.

Tendo-se em vista a escassez de estudos clínicos com plantas medicinais é importante considerar que algumas plantas utilizadas podem ter efeitos tóxicos, como hepatotoxidade e bloqueio $\beta$ - adrenérgico, os quais podem resultar no efeito desejado hipoglicemia, mas não como fenômeno terapêutico e sim tóxico. Isto enfatiza a necessidade de encontrar aquelas que possam oferecer eficácia terapêutica e saúde. A toxicidade ainda pode ser influenciada pela parte da planta usada na preparação do extrato, método de preparação e rota de administração (Negri, 2005).

No presente trabalho houve preocupação especial relacionada ao modo de preparo destas plantas medicinais, assim como quanto ao acondicionamento do preparado, estabilidade, interações com alimentos e fármacos hipoglicemiantes, doses utilizadas e posologias, o que pode subestimar o tratamento preterido ou elevar os riscos para toxicidade de espécies cujos efeitos terapêuticos e tóxicos estão pouco esclarecidos nos seres humanos.

Infelizmente, a maior parte dos fitoterápicos e plantas medicinais que são utilizados atualmente por automedicação ou por prescrição médica não tem o perfil tóxico bem conhecido (Capasso et al., 2000; Veiga Júnior, 2008). Por outro lado, a utilização inadequada de produto, mesmo de baixa toxicidade, pode induzir problemas graves desde que existam outros fatores de risco tais como contra-indicações ou uso concomitante de outros medicamentos (Coelho, 1998; Cordeiro et al., 2005; Amorim et al., 2007).

Como as pesquisas para avaliação da segurança de plantas medicinais e fitoterápicos no Brasil ainda são escassas, faz-se necessário controle rígido da comercialização da venda desses produtos em feiras, mercados ou lojas de produtos naturais, na tentativa de reduzir erros de identificação da planta (pelo comerciante e/ou comprador), possibilidades de adulteração dos compostos derivados das plantas, reduzindo os riscos de superdosagem, adulterações nos produtos e reações alérgicas ou tóxicas (Veiga Júnior et al., 2005).

A prática do uso de plantas medicinais deve ser fortalecida por estudos que comprovem a eficácia em seres humanos, contribuindo para estabelecer o perfil de toxicidade e uso a longo prazo em pacientes diabéticos, com acompanhamento da evolução das complicações, tais como neuropatia periférica, fenômenos micro e macrovasculares e nefropatia. Estes parâmetros podem encorajar o uso tradicional destas espécies ou diminuir a exposição da população à práticas pouco seguras.

Neste aspecto, é de fundamental importância a criteriosa avaliação dos fenômenos farmacoeconômicos que guiam a necessidade de implantação dos programas farmácia viva, assim como o suporte adequado para funcionamento destes programas com foco especial na formação dos profissionais de saúde e de agentes multiplicadores que atuem diretamente na educação da população 
com relação ao uso responsável das plantas medicinais, com eficaz monitoramento da farmacovigilância de plantas medicinais e agentes fitoterápicos.

O cadastramento das farmácias vivas em funcionamento no país, assim como a estruturação e auxílio na manutenção de programas de referência, fontes inesgotáveis de conhecimento e formação, são ações sugeridas. A catalogação e uso de plantas medicinais para tratamento de diabetes que possuam certificação e estudos de constituintes fitoquímicos e efeitos farmacológicos são passos fundamentais na realização de futuros estudos em seres humanos, que mesmo dispendiosos durante a fase de ensaios, podem representar uma alternativa preciosa no controle do diabetes e outras patologias crônicas para a população de um país continental como o Brasil, garantindo o acesso desta população a um tratamento responsável e ajustando os gastos governamentais na garantia deste fenômeno e promoção da saúde.

As três plantas medicinais mais amplamente utilizadas pelos pacientes avaliados foram pata-devaca (Bahuinia sp), azeitona roxa (Syzygium jambolanum DC.) e insulina (Cissus sicyoides L.), espécies já amplamente estudadas e com efeitos hipoglicemiantes elucidados pela comunidade científica, o que corrobora o uso no tratamento de diabetes por esta população.

\section{REFERÊNCIA}

ALMEIDA, R.N. Avaliação da atividade hipoglicemiante e isolamento de alguns triterpenóides de Bumelia sartorum Mart. 1982. 183p. Dissertação (Mestrado) Universidade Federal da Paraíba, João Pessoa.

AMORIM, M.F.D. et al. The controvertible role of kava (Piper methysticum G. Foster) an anxiolytic herb, on toxic hepatitis. Revista Brasileira Farmacognosia, v.17, n.3, p.448-54, 2007.

ARAÚJO, A. M. Medicina rústica. 2.ed. São Paulo: Ed. Nacional, 1977. 396p.

ARDUINO, F.H.; SOARES, M.L. Ação hipoglicemiante do cajueiro em indivíduos normais. Brasil Médico, v.65, p.305-8, 1951.

ASSUNÇÃO, M.C.F.; SANTOS, I.S.; COSTA, J.S.D. Avaliação do processo da atenção médica: adequação do tratamento de pacientes com Diabetes mellitus, Pelotas, Rio Grande do Sul, Brasil. Cadernos de Saúde Pública, v.18, n.1, p.205-11, 2002.

BALUCHNEJADMOJARAD, T. et al. Beneficial effect of aqueous garlic extract on the vascular reactivity of streptozotocin diabetic rats. Journal Ethnopharmacology, v.85, n.1, p.139-44, 2003.

BATALHA, M.O. et al. Plantas medicinais no Estado de São Paulo: situação atual, perspectivas e entraves ao desenvolvimento. Florestar Estatístico, v.6, n.15, p. 2735, 2003.

BORGES, K.B.; BAUTISTA, H.B.; GUILERA, S. Diabetes - utilização de plantas medicinais como forma opcional de tratamento. Revista Eletrônica de Farmácia, v.5, n.2, p.12-20, 2008.

BRAGANÇA, L.A.R. Plantas medicinais antidiabéticas. Uma abordagem multidisciplinar. Rio de Janeiro: Editora EDUFF, 1996. 300p.

BRASIL. Ministério da saúde. Inquérito Domiciliar sobre Comportamento de Risco e Morbidade Referida de Doenças e Agravos não Transmissíveis: Brasil, 15 capitais e Distrito Federal. 2003, p.141-9. Disponível em: <http://www.inca.gov.br/inquerito/docs/introd>. Acesso em: 24 ago. 2010.

BRITO, S.C.D. Os efeitos do marco regulatório sobre a competitividade da cadeia produtiva de medicamentos fitoterápicos no Brasil. 2010. 96p. Dissertação (Mestrado em desenvolvimento regional e Agronegócio)Fundação Universidade Federal de Tocatins, Palmas. CAPASSO, R. et al. Phytotherapy and quality of herbal medicines. Fitoterapia, v.71, p.S58-S65, 2000.

CARVALHO, A.C.B.; DINIZ, M.F.F.M.; MUKHERJEE, R. Estudos da atividade antidiabética de algumas plantas de uso popular contra o diabetes no Brasil. Revista Brasileira de Farmácia, v.86, n.1, p.11-6, 2005.

CECÍLIO, A.B. et al. Espécies vegetais indicadas no tratamento do diabetes. Revista Eletrônica de Farmácia, v.5, n.3, p.23-8, 2008.

COELHO, H.L. Farmacovigilância: um instrumento necessário. Cadernos de Saúde Pública, v.14, n.4, p.871-5, 1998.

CONFERÊNCIA NACIONAL DE SAÚDE. CONFERÊNCIA NACIONAL DE SAÚDE. 5., 1975. Brasília. Resumos... Brasília: Ministério da Saúde, 1975. 355p.

CORDEIRO, C.H.G.; CHUNG, M.C.; SACRAMENTO, L.V.S. Interações medicamentosas de fitoterápicos e fármacos: Hypericum perforatum e Piper methysticum. Revista Brasileira de Farmacognosia, v.15, n.3, p.2728, 2005.

CÓRDOVA, K.V. et al. Características físico-químicas da casca do maracujá amarelo (Passiflora edulis flavicarpa Degener) obtida por secagem. Boletim do Centro de Pesquisa de Processamento de Alimentos, v.23, n.2, p.221-30, 2005.

CORREA M.P. Dicionário de plantas úteis do Brasil e das exóticas cultivadas. Rio de Janeiro: Ministério da Agricultura, 1984. 172p.

COSTA, C.M.M. Cipó-pucá (Cissus sycioides). Rio de Janeiro, Apostila do curso de Especialização em Medicamentos da UFRJ, 1990. 109p.

DAB - Departamento de Atenção Básica, Secretaria de Políticas de Saúde. Programa Saúde da Família. Revista de Saúde Pública, v.34, p.316-9, 2000.

DERIVI, S.C.N. Efeito hipoglicêmico de rações à base de berinjela (Solanum melongena L.) em ratos. Ciência e Tecnologia de Alimentos, v.22, n.2, p.164-9, 2002. DIEGUES, A.C.S. O mito moderno da natureza intocada. 2.ed. São Paulo: Hucitec, 1996. 169p.

ELIZABETSKY, E. Ação anticonvulsivante de Cissus sycioides, cipó-pucá. Ciência e Cultura, v.40, n.7, p.985, 1988.

FUENTES, O.; ARANCIBIA-AVILA P.; ALARCON J. Hypoglycemic activity of Bauhinia candicans in diabetic induced rabbits. Fitoterapia, v.75, n.6, p.527-32, 2004. GARCIA, E.S. Biodiversity, biotechnology and health.

Rev. Bras. PI. Med., Botucatu, v.14, n.2, p.327-334, 2012. 
Cadernos de Saúde Pública, v.11, n.3, p.495-500, 1995. GROVER, J.K.; VATS, V.; RATHI, S.S. Anti-hyperglicemic effectof Eugenia jambolana and Tinospora cordifolia in experimental diabetes and their key metabolic enzymes involved in carbohydratemetabolism. Journal of Ethnopharmacology, v.73, p.461-70, 2000.

JAFRI, M.A. et al. Effect of Punica granatum Linn. (flowers) on blood glucose level in normal and alloxan - induced diabetic rats. Journal of Ethnopharmacology, v.70, p.30914, 2000.

KRAHN C.L. et al. Avaliação do efeito da casca desidratada do maracujá (Passiflora edulis) e seu extrato aquoso na redução da glicemia em ratos diabéticos induzidos por aloxano. Revista Brasileira de Farmácia, v.89, p.32-4, 2008.

LINO, C.S. et al. Antidiabetic activity of Bauhinia forficata extracts in alloxan-diabetic rats. Biological \& Pharmaceutical Bulletin, v.27, n.1, p.125-7, 2004.

MACEDO, M.; FERREIRA, A.R. Plantas hipoglicemiantes utilizadas por comunidades tradicionais na Bacia do Alto Paraguai e Vale do Guaporé, Mato Grosso-Brasil. Revista Brasileira de Farmacognosia, v.14, p.45-7, 2004. MARLES, R.J.; FARNSWORTH, N.R. Antidiabetic plants and their active constituents. Review. Phytomedicine, v.2, p.137-89, 1995.

MOURA, B.A.S. Estudo químico e farmacológico de espécie vegetal Cissus sycioides Linn. (Apostila do curso de especialização em química de produtos naturais da Universidade do Pará), Belém, 1986. 98p. MS - Ministério da Saúde. A implantação da Unidade de Saúde da Família. Brasília: Secretaria de Políticas de Saúde, Ministério da Saúde, 2000. 44p.

MS - Ministério da Saúde. Política Nacional de Plantas Medicinais e Fitoterápicos. Secretaria de Ciência, Tecnologia e Insumos Estratégicos - Departamento de Assistência Farmacêutica. p.60. Distrito Federal, 2006. NAIK, S.R. et al. Probable mechanism of hypoglycemic activity of bassic acid, a natural product isolated from Bumelia sartorum. Journal of Ethnopharmacology, v.33, n.1-2, p.37-44, 1991.

NOGUEIRA, D.G.; PEREIRA, N.A. Atividade inibidora da absorção intestinal da glicose do epicarpo da romã (Punica granatum L.). Revista Brasileira de Farmácia, v.67, p.129-34, 1986.

NEGRI, G. Diabetes melito: plantas e princípios ativos naturais hipoglicemiantes. Revista Brasileira de Ciências Farmacêuticas, v.41, n.2, p.121-42, 2005.

PAIVA, D.C.P. et al. Avaliação da assistência ao paciente com diabetes e/ou hipertensão pelo Programa Saúde da Família do Município de Francisco Morato, São Paulo, Brasil. Caderno de Saúde Pública, v.22, p.377-85, 2006. PEPATO, M.T. Efeito da administração crônica de Cissus sicyoides no metabolismo de carboidratos. In: SIMPÓSIO BRASILEIRO DE PLANTAS MEDICINAIS, 15., 1998, Águas de Lindóia, SP. Resumo .... São Paulo: Sociedade Brasileira de Plantas Medicinais, 1998, p.78.
PEPATO, M.T. et al. Anti-diabetic activity of Bauhinia forficata decoction in stretozotocin-diabetic rats. Journal of Ethnopharmacology, v.81, n.2, p.191-7, 2002.

PEPATO, M.T. et al. Evaluation of toxicity after one-months treatment with Bauhinia forficata decoction in stretozotocin-induced diabetic rats. Biomed Central Complementary and Alternative Medicine, v.4, p.1-7, 2004.

PRINCE, P.S.M.; KAMALAKKANNAN, N.; MENON, V.P. Syzigium cumini seed extracts reduce tissue damage in diabetic rat brain. Journal of Ethnopharmacology, v.84, p.205-9, 2003.

RAMOS, E.R.F. O uso de Passiflora sp. no controle do Diabetes mellitus: estudo qualitativo preliminar. 2004. 36p. Monografia (Monografia de Conclusão de Curso de Farmácia) - Centro Universitário de Maringá, Maringá. RAO, B.K.; KESAVULU, M.M.; APPARAO, C.H. Antihyperglicemic activity of Momordica cymbalaria in alloxan diabetic rats. Journal of Ethnopharmacology, v.78, p.67-71, 2001.

RATHI, S.S. et al. Prevention of experimental diabetic cataract by Indian Ayurvedic plant extracts. Phytotherapy Research, v.16, p.774-7, 2002.

REZENDE, H.A.; COCCO, M.I.M. A utilização de fitoterapia no cotidiano de uma população rural. Revista da Escola de Enfermagem, v.36, n.3, p.282-8, 2002.

SHIRWAIKAR, A. et al. Antidiabetic activity of aqueous leaf extract of Annona squamosa in streptozotocinnicotinamide type 2 diabetic rats. Journal of Ethnopharmacology, v.91, p.171-5, 2004.

SILVA, M.B.; MENDEZ, M.H.M.; DERIVI, S.C.N. Efeito hipoglicêmico de alimentos ricos em fibra solúvel. Estudo com o jiló (Solanum gilo Raddi). Alimentos e Nutrição, v.9, p.43-64, 1998.

SOARES, J.C.M.; COSTA, S.T.; CECIM, M. Níveis glicêmicos e de colesterol em ratos com Diabetes mellitus aloxano induzido, tratados com infusão de Bauhinia candicans ou Syzygium jambolanum. Ciência Rural, v.30, p.113-8, 2000.

VEIGA JUNIOR, V.F.; PINTO, A.C.; MACIEL, M.A.M. Plantas medicinais: cura segura? Quimica Nova, v.28, n.3, p.51928, 2005.

VEIGA JUNIOR, V.F. Estudo do consumo de plantas medicinais na Região Centro-Norte do Estado do Rio de Janeiro: aceitação pelos profissionais de saúde e modo de uso pela população. Revista Brasileira de Farmacognosia, v.18, p.308-13, 2008.

WORLD HEALTH ORGANIZATION. Diabetes. Fact sheet № 312, Novembro de 2009. Disponível em: <http:// www.who.int/mediacentre/factsheets/fs $312 / \mathrm{en} /$ index.html>. Acesso em: 30 nov. 2010

ZANOELLO, A.M. et al. Efeito protetor do Syzygium cumini contra Diabetes mellitus induzido por aloxano em ratos. Acta Farmaceutica Bonaerense, v.21, n.1, p.31-6, 2001. 